\title{
Be aware although it's rare! Toxic Shock Syndrome following Endoscopic Sinus Surgery and Septoplasty
}

\author{
S Naidoo, O Edkins \\ Department of ENT, New Somerset Hospital, Cape Town \\ Corresponding author: Shavina Naido (shavina.naidoo@gmail.com)
}

Summary: This case report illustrates the rapid and fulminant course of Staphylococcal Toxic Shock Syndrome as a potential postoperative complication of sino-nasal surgery, and serves as an aid to otorhinolaryngologists for its recognition and early goal-directed therapy.

S Afr J Surg 2018;56(3)

http://dx.doi.org/10.17159/2078-5151/2017/v56n3a2394

\section{Introduction}

Toxic Shock Syndrome (TSS) is a rare and potentially lifethreatening complication of Endoscopic Sino-nasal Surgery. Its incidence is reported in up to 16 per 100,000 cases $^{1}$ and it usually affects well, young individuals.

There are multiple aetiological factors that need to be present to develop TSS: the colonisation of the nasal cavity by Staphylococcus aureus (S. aureus), a breach in the mucosal surface, haematogenous spread of the organism and a lowered host defence, namely the lack of anti-toxin antibodies. ${ }^{1,2,3}$ $S$. aureus produces super antigens, TSST-1, or enterotoxinlike toxin, that trigger an exaggerated immune response with excessive cytokine release and T-cell activation. ${ }^{3}$ This results in rapid onset of an influenza-type illness and multi-systemic dysfunction. ${ }^{4}$ The diagnostic criteria are mostly based on comparable clinical findings (Table 1) as only up to $5 \%$ of blood cultures and nasopharyngeal swabs are positive for $S$. aureus in the presence of TSS. ${ }^{5,6}$

This case report highlights the rapid decline of a young, healthy individual who underwent routine, elective endoscopic sinus-nasal surgery.

\section{Case Report}

A healthy 21-year-old male, with no known medical comorbidities, underwent an elective limited endoscopic

Table 1: Case Definition of Toxic Shock Syndrome ${ }^{5,6}$

\begin{tabular}{ll}
\hline Requires 5 or more to be present: & Case Patient: \\
\hline a. Fever: $>38.9^{\circ} \mathrm{C}$ & Positive \\
b. Rash: Diffuse, erythroderma and desquamation of soles and palms (1-2weeks post onset of illness) & Positive \\
c. Hypotension: Systolic BP of $<90 \mathrm{mmHg}$ in adults & Positive \\
d. Multi-Systemic Involvement: 3 or more present & Positive \\
1. Gastrointestinal - Vomiting/ Diarrhoea & Positive \\
2. Muscular - myalgia or raised serum CK & Positive \\
3. Mucus membranes - hyperaemia of oropharynx/conjunctivae/ vaginal & Negative \\
4. Renal - raised serum renal functions & Positive \\
5. Hepatic - raised serum bilirubin & Negative \\
6. Haematological - platelets $<100 X 10^{9} \mathrm{~L}^{-1}$ & \\
7. Neurological - delirium/disorientation with no focal signs & Negative \\
e. Negative Test Results & Did not test \\
Other Organisms: Blood/Throat Culture/Cerebrospinal Fluid & \\
Raised levels of Rocky Mountain Spotted Fever or Leptospirosis or Measles &
\end{tabular}


sinus surgery and septoplasty for chronic rhinosinusitis and a deviated nasal septum respectively. He required no nasal packing during the peri- or postoperative period. No prophylactic antibiotics were given.

He developed symptoms of fever, rigors, vomiting, diarrhoea, hypotension, and tachycardia less than 12 hours post-surgery. On physical examination, the patient had diffuse, erythroderma and abdominal tenderness. An initial differential diagnosis of septic shock, acute allergic reaction, and acute infective gastroenteritis was made.

Aggressive intravenous resuscitation with crystalloids was started and, being oliguric, a urinary catheter was inserted to measure urine output. Invasive haemodynamic monitoring was also established (central venous line and arterial line) and inotropic support was commenced after admission to the intensive care unit (ICU). Routine bloods (full blood count, electrolytes, and renal function), blood cultures and a pus swab of the oropharynx were taken. After transfer to ICU, he received care from a multidisciplinary team, including internal medicine, intensive care physicians, infectious diseases, and the otorhinolaryngologists who had performed the surgery.

Blood results showed that he had acute renal injury, electrolyte disturbances, low platelets and a metabolic acidosis. The stool sample tested positive for $C$. difficile, despite only being admitted to hospital for 24-48 hours at the time of the test, and a throat swab was positive for $S$. aureus. Triple intravenous antibiotics were commenced: Cloxacillin, Metronidazole, and Ceftriaxone, and he was discharged from ICU on day 2 post-surgery.

On the third day, post-surgery, the patient developed Acute Respiratory Distress Syndrome with bilateral effusions shown on chest tomography. A pleural tap of the chest was done for the pleural effusions and continuous oxygen therapy given. He required physiotherapy, and anti-thrombotic treatment was continued. At day 5 post-surgery, the patient showed much improvement and by day 7 post-surgery, the patient was discharged from hospital with a continued course of oral antibiotics. At his one-month follow-up, with both the internal medicine physicians and ENT surgeons, there were no residual effects of the illness and his nasal symptoms continued to improve.

\section{Discussion}

Nasal carriage of $S$. aureus is an important endogenous risk factor for many patients undergoing nasal surgery. ${ }^{1} S$. aureus colonisation of the upper airway, skin, and genital tract is common, with $27 \%$ of the general population being persistent nasal carriers and as much as $60 \%$ being intermittent carriers. ${ }^{7}$ Of these nasal carriers (intermittent and persistent), 30\% have the potential to produce super antigens. ${ }^{2}$

Once multiple factors are at play, the patient quickly becomes severely ill and requires prompt recognition of the syndrome and decisive management within hours of presentation of illness. This multi-modality management is outlined by_The International Guidelines for Management of Severe Sepsis and Septic Shock, 2008. ${ }^{6}$ Special attention is given to aggressive fluid resuscitation and inotropic support in ICU and prompt, targeted antibiotic therapy, dependant on local regimens and resistance rates. Other adjunctive treatments are described, but are not necessary for every patient presenting with TSS.

In our case report, despite the health status of the patient and the elective, uncomplicated surgery, the patient presented with more than five of the necessary symptoms required by the case definition. He was promptly recognised as a potential septic shock case and transferred to ICU. Immediate intravenous support and invasive monitoring was started and the hospital antibiotic regimen for septic shock started. In our case, the throat swab was positive for $S$. aureus. He recovered relatively quickly and today has no sequel from his experience.

In hindsight, it is difficult to say if any preventative measures should have been in place to prevent TSS. According to the literature, TSS is a rare complication of $S$. aureus infection ${ }^{8}$ and requires many factors to be present for its development. The key factor in this case presentation, is that the condition was recognised and management of it initiated without much delay.

In many studies, the elimination of nasal carriage seems to reduce the infection rates of surgical patients. ${ }^{1}$ Multiple treatment modalities, pre- and postoperative prophylactic systemic antibiotics or colonisation of the nose with less virulent strains of $S$. aureus, were tested. ${ }^{9}$ Many showed little or no change in infection rates as compared with control groups, with some demonstrating higher rates of organism resistance

The International Guidelines for Management of Severe Sepsis and Septic Shock, $2008^{6}$

\begin{tabular}{|l|l|l|l|l|l|}
\hline & & & $\begin{array}{l}\text { Aggressive fluid } \\
\text { resuscitation }\end{array}$ & $\begin{array}{l}\text { Inotropic support } \\
\text { and invasive } \\
\text { haemodynamic } \\
\text { monitoring }\end{array}$ \\
$\begin{array}{l}\text { Surge response of } \\
\text { Immune System } \\
\text { to Super Antigens }\end{array}$ & $\begin{array}{l}\text { Rapid onset of } \\
\text { symptoms and } \\
\text { establishment of } \\
\text { septic shock }\end{array}$ & $\begin{array}{l}\text { Prompt } \\
\text { recognition of } \\
\text { the syndrome } \\
\text { and decisive } \\
\text { management }\end{array}$ & $\begin{array}{l}\text { Targeted } \\
\text { antibiotic therapy, } \\
\text { dependant on } \\
\text { local regimens } \\
\text { and resistance } \\
\text { rates }\end{array}$ & $\begin{array}{l}\text { Adjunctive } \\
\text { therapies: Human } \\
\text { Immunoglobulin } \\
\text { or Activated } \\
\text { Protein C. }\end{array}$ \\
\hline
\end{tabular}


and, with bacterial interference, serious complications. There was also no statistically significant correlation found between nasal swab positive patients for $S$. aureus or the positive testing of TSST-1 polymerase antigen and the development of TSS. Currently, the only drug showing any promise is Mupirocin (nasal ointment preparation), with one multicentre study done in 1993, showing a 91\% elimination rate as compared to placebo groups. However, due to the prolonged skin preparation time and high risk of resistance, as shown by its predecessor, Naseptin, in many other studies, its routine use is limited..$^{5,7}$

\section{Conclusion}

Routine prophylactic antibiotic use both systemically or intra-nasally, or routine testing of every patient coming for endoscopic nasal surgery is not warranted, according to current research outcomes. The key to combating TSS is awareness on the part of clinicians and early recognition of the syndrome with immediate implementation of resuscitative measures and timeous appropriate level of care thereafter.

\section{REFERENCES:}

1. Erisir F, Inci E. Toxic Shock Syndrome Following Endoscopic Surgery. Turk Arch Otolaryngol. 2001;39(4):305-7.

2. Meyers AD. Prophylactic Antibiotics in Nasal Surgery. Arch
Otolaryngol Head Neck Surg. 1990;116(10):1125-6.

3. Peril TM, Cullen JJ, Wenzel RP, Zimmerman MB, Pfaller MA, Sheppard D, et al. Intranasal Mupirocin to Prevent Postoperative Staphylococcus aureus Infections. N Eng J Med. 13 June 2002;346(24):1871-7.

4. Silversides J, Lappin E, Ferguson A. Staphylococcal Toxic Shock Syndrome: Mechanisms and Management. Curr Infect Dis Rep. June 2010;12(5):392-400. doi: 10.1007/ s11908-010-0119-y

5. Darenberg J, Soderquist B, Normark BH, Norrby-Teglund A. Differences in potency of intravenous polyspecific immunoglobulin G against streptococcal and Staphylococcal superantigens: implications for therapy of toxic shock syndrome. Clin Inf Dis. 2004;38:836-42.

6. Centers for Disease Control and Prevention Toxic Shock Syndrome. Case definition. 1997 [accessed on 5 Oct 2005]. Available from: http://www.cdc.gov/epo/dphsi/casedef/ toxicsscurrent.htm

7. Göçer C, Dursun E, Acar A, Özman M, Dağli M, Balaban N, et al: Antimicrobial Prophylaxis in Nasoseptal Surgery. KBBForum. 2007;6(1):10-14.

8. Kluytmans J, van Belkum A, Verbrugh H: Nasal carriage of Staphylococcus aureus: epidemiology, underlying mechanisms, and associated risks; Clin Microbiol Rev. July 1997;10(3):50520.

9. Jacobson JA, Stevens MH, Kasworm E. Evaluation of singledose cefazolin prophylaxis for toxic shock syndrome. Arch Otolaryngol Head Neck Surg. 1988;114(3):326-7. doi: 10.1001/ archotol.1988.01860150108025 